\title{
Direct observation of crystallization and aggregation in a phase-separating colloid-polymer suspension
}

\author{
E. H. A. de Hoog, ${ }^{1}$ W. K. Kegel, ${ }^{1}$ A. van Blaaderen, ${ }^{2,3}$ and H. N. W. Lekkerkerker, ${ }^{1, *}$ \\ ${ }^{1}$ Van 't Hoff Laboratory for Physical and Colloid Chemistry, Debye Institute, Utrecht University, Padualaan 8 , \\ 3584 CH Utrecht, The Netherlands \\ ${ }^{2}$ FOM-Institute for Atomic and Molecular Physics, Kruislaan 407, 1098 SJ Amsterdam, The Netherlands \\ ${ }^{3}$ Physics and Chemistry of Condensed Matter, Debye Institute, Utrecht University, Princetonplein 5 , \\ 3584 CC Utrecht, The Netherlands
}

(Received 28 February 2001; published 26 July 2001)

\begin{abstract}
The depletion-induced phase separation in a mixture of colloidal particles (PMMA-latex) and nonadsorbing polymers [poly(styrene)] in a solvent (mixture of tetralin, cis-decalin, and carbon tetrachloride) was investigated in real space with confocal scanning laser microscopy in the initial, intermediate, and final stage. It was found that the kinetics and the morphology of the phase separation strongly depend on the polymer concentration, and thus on the strength of the depletion-induced attraction between the colloidal particles. At moderate polymer concentrations, crystallization of the PMMA particles is enhanced. At higher polymer concentrations, only aggregation is observed, resulting in amorphous sediments. The aggregation is diffusion-limited or reaction-limited, depending on the polymer concentration. Digital image processing was used to determine the dependence of the aggregation rate and the size of the clusters on the polymer concentration.
\end{abstract}

DOI: 10.1103/PhysRevE.64.021407

PACS number(s): 82.70.Dd, 64.70.-p, 61.43.Hv

\section{INTRODUCTION}

Phase separation in colloid-polymer suspensions is attracting wide attention. The subject ranges from fundamental studies in colloid science and cell biology to industrial applications. It is now generally accepted that phase separation in mixtures of colloidal particles and nonadsorbing polymers is induced by depletion interaction. The basic concept dates back to 1954, when Asakura and Oosawa [1] showed that an effective attractive interaction is induced between two larger particles in a solvent with smaller particles, because of an imbalance in osmotic pressure due to depletion of the smaller particles from the region between the larger ones. This idea was put forward and expanded independently by Vrij [2] in 1976. The range of the attractive interaction is set by the size of the polymer and its strength depends on the osmotic pressure, which in turn is a function of the polymer concentration. The phase behavior of colloid-polymer suspensions has been studied theoretically, experimentally, and with computer simulations [3-9]. The topology of the phase diagram of these systems depends on the size ratio, $q$, defined as the ratio of the polymer radius of gyration and the colloid radius. The existence of fluid-fluid and fluid-solid phase transitions has been predicted by theory $[3,4]$ and confirmed by experiments $[6,7]$.

More recently, interest has been growing in the kinetics of these phase transitions $[8,10,11]$. Glasses, gels, and amorphous structures were described in experiments with colloidal suspensions. An interesting observation is that in fluidsolid transitions, the solid phase is sometimes a crystal and sometimes an amorphous structure. In earlier studies, a narrow window of crystallization was observed. Near the bin-

\footnotetext{
*Corresponding author. Email address: H.N.W.Lekkerkerker@chem.uu.nl
}

odal, at a minimum concentration of depletion agents required for phase separation, a small region of optimal crystallization is observed. The addition of more depletion agents increases the phase-separation rate, but does not lead to crystallization.

First, the narrow crystallization slot was found by visual observation $[10,12,13]$. Subsequently, light scattering experiments gave more insight into the kinetics and structures (e.g., transient gels [10]) involved in the phase-separation process of colloid-polymer suspensions $[8,11]$. With the development of (video) microscopy techniques and the synthesis of model colloidal systems, the study of aggregation and crystallization on the particle level became possible. With optical microscopy, two-dimensional aggregation on surfaces or in confined geometries was studied [14,15]. More recently, fluorescence confocal scanning laser microscopy came into use to study statics [16] and dynamics of colloidal suspensions, such as dynamical heterogeneities [17], structural relaxation near the glass transition [18], and crystallization [19] in colloidal hard-sphere suspensions. The use of fluorescence confocal scanning laser microscopy (CSLM) in principle enables imaging the three-dimensional process in real time and real space on a single-particle level, not only in the initial stage but also at long time scales, which are generally not easily accessible with computer simulations.

In this paper, we investigate the kinetics and morphology of phase separation as a function of the strength of attraction between the particles. A colloid-polymer suspension is used as a model system and the use of fluorescence CSLM allows us to study it in real space and real time on a "semisingle" particle level. The strength between the colloidal particles is varied by the polymer concentration. The experiments are carried out at low colloid concentrations, because the dynamics are too fast at higher colloid concentrations to investigate the initial stage with the microscope used. The colloid con- 
centration in the samples is thus fixed, while the polymer concentration is varied.

The paper is organized as follows. In the experimental section, the system and the microscopy experiments are described. In the following section, the determined phase diagram and the results of the microscopy experiments are given. Subsequently, in the analysis the results of the digitalimage processing are presented. Then a discussion on the experiments is given, followed by the conclusions.

\section{EXPERIMENTAL SECTION}

\section{A. System}

The colloidal particles used were PMMA particles (polymethylmethacrylate) prepared by dispersion polymerization at $80^{\circ} \mathrm{C}$, following the method of Antl [20]. The particles were labeled with the fluorescent dye 7-nitrobenzo2-oxa-1,3-diazol (NBD) bonded to a coupling agent (4-methylaminoethylmethacrylate-7-nitrobenzo-2-oxa-1,3diazol), which was incorporated during the polymerization reaction. The particles are stabilized by a layer of $10 \mathrm{~nm}$ of a graft copolymer consisting of a backbone of methacrylate and glycidyl methacrylate monomers in a weight ratio of 10:1, and tails of poly(12-hydroxystearic acid) are attached to some of the glycidyl methacrylate monomers. The stabilizer is chemically bonded by a locking reaction at $130^{\circ} \mathrm{C}$. The synthesis of these particles is described elsewhere in more detail [21]. The radius was determined with both CSLM and scanning electron microscopy, and was found to be 600 and $580 \mathrm{~nm}$, respectively, with a polydispersity of $3 \%$. The polymer used is commercially available poly(styrene); its molecular weight is $2000 \mathrm{~kg} / \mathrm{mol}\left(M_{w} / M_{n}\right.$ $=1.11$ ), and its density is $1.11 \mathrm{~g} / \mathrm{ml}$. The viscosity of solutions of this polymer in the solvent mixture was measured as a function of the polymer concentration using a Contraves LS40 rheometer with a Couette geometry equipped with a vapor lock. The radius of gyration, determined from these viscosity measurements [22], is $46 \mathrm{~nm}$, which implies an overlap concentration of $8.15 \mathrm{mg} / \mathrm{ml}$. Here, the polymer volume fraction is defined as (4/3) $\pi n R_{g}^{3}$, with $n$ the number density of the polymers and $R_{g}$ the radius of gyration. The solvent used was a mixture of tetralin, cis-decalin, and carbon tetrachloride in volume ratios of $36 \%$ (30.3 wt. \%), $31.5 \%$ (24.6 wt. \%), and $32.5 \%$ (45.1 wt. \%), respectively; between brackets the weight ratios are given. This particular solvent mixture was chosen after a study of the stability and behavior of the PMMA particles in various solvents as described elsewhere [23]. The density is $1.148 \mathrm{~g} / \mathrm{ml}$, the index of refraction is 1.50 , and the viscosity is $1.87 \mathrm{mPa}$. The density of the PMMA particles in this solvent mixture is 1.25 $\mathrm{g} / \mathrm{ml}$, as determined from the sedimentation rate. In this solvent mixture, the PMMA particles are stable for a long time (at least several months), they are nearly matched for the index of refraction, but not completely matched for the density (density difference is on the order of $0.1 \mathrm{~g} / \mathrm{ml}$ ). The PMMA particles are behaving as nearly hard spheres, since the index matching minimizes interparticle attractions due to Van der Waals forces, and the PHS layer provides a steep

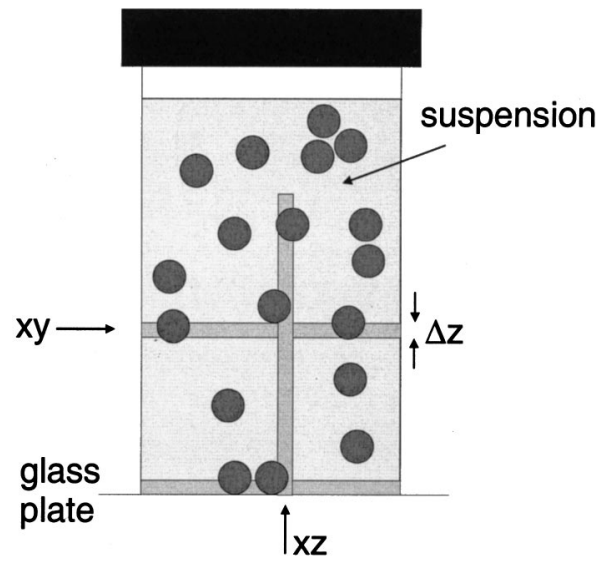

FIG. 1. Sample container used in CSLM. The positions of the $x y$ and the $x z$ scans are indicated.

repulsive potential arising from interpenetration of these polymer layers of different particles upon approach.

\section{B. Microscopy}

A Leica confocal scanning laser microscope in fluorescence mode was used [19], the objective was a $100 \times$ oil lens with a numerical aperture of 1.4, the wavelength of excitation was $488 \mathrm{~nm}$, a filter block for detection of light of wavelengths larger than $500 \mathrm{~nm}$ was used, and the time needed to take an image of $100 \mu \mathrm{m} \times 100 \mu \mathrm{m}$ with $1024 \times 1024$ pixels was $3 \mathrm{~s}$. The lateral resolution of the microscope is about 200 $\mathrm{nm}$ and the axial resolution is about $600 \mathrm{~nm}$. The sample was contained in a small vial (volume of $\sim 1 \mathrm{ml}$ ) from which the bottom was replaced by a microscope cover slip (Chance Propper Ltd., West Mids, England) of 0.11-mm thickness. The vial was filled with $0.3 \mathrm{ml}$ of dispersion and sealed with teflon. Images were taken at $20 \mu \mathrm{m}$ from the lower wall, a distance that equals $\sim 20$ times the diameter of the colloids. The influence of the wall on the process is considered negligible at this position. The three-dimensional (3D) process is studied with CSLM in 2D cross sections of the sample. The CSLM images shown in this paper are either $x y$ scans, i.e., cross sections perpendicular to the field of gravity, or $x z$ scans, which are cross sections parallel to the field of gravity; see Fig. 1.

\section{RESULTS}

\section{A. Phase diagram}

After homogenization of a sample at sufficiently high concentration of polymers, phase separation is observed within an hour by a sedimentation front between a colloidpoor upper phase (light yellow) and a colloid-rich lower phase (dark yellow) and the appearance of a sediment at the bottom. The phase boundary was determined by visual inspection of suspensions with concentrations varying along dilution lines (i.e., straight lines through the phase diagram with constant concentration ratio of colloid and polymer), starting at high concentrations. It was observed that on approaching the phase boundary by dilution, the sedimentation 


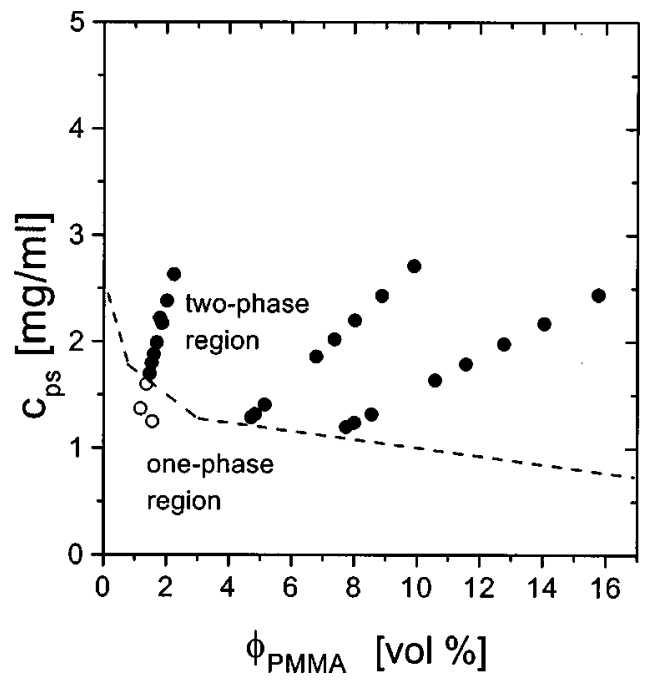

FIG. 2. Phase diagram of the colloid-polymer suspension with the one-phase and the two-phase regions indicated. To guide the eye, the phase boundary is indicated with a dashed line.

velocity lowers and it became difficult to distinguish between phase separation and sedimentation of the particles in a single phase. In Fig. 2, a phase diagram is shown in which the one-phase and the two-phase regions are indicated. At the higher colloid concentration regions, the visual inspection along the dilution line is stopped close to the phase boundary, at a point where we were still certain to have a phaseseparating system. The phase boundary is just below these last points.

The structure of the sediment was examined visually in a few samples with a polymer concentration ranging from zero to a concentration just sufficiently high to show phase separation. The samples were prepared in "sedimentation" tubes with an internal diameter of $6 \mathrm{~mm}$ and a round bottom. Each tube was filled with $1 \mathrm{ml}$ of suspension. Note that these tubes are larger and have a different shape from the vials used for the CSLM experiments. We found that the structure formed was dependent on the polymer concentration. At zero polymer concentration, the whole sediment turned out to be a crystal with vertically oriented crystal planes, shown by its Bragg reflections. The crystal started to form within a few days. At higher polymer concentrations, but still below the phase boundary, a crystal was observed only at the upper side of the sediment, after about a week. This structure did not show vertically oriented crystal planes, but consisted of smaller crystalline domains. At even higher polymer concentrations, just above the phase boundary, the crystal layer was smaller. The sediment of the phase-separated sample showed only some very small crystal domains on top of the sediment.

\section{B. Microscopy}

A series of 20 samples was made with a fixed PMMA volume fraction of $2.2 \%$ and a varying concentration of poly(styrene) ranging from 0 up to $9 \mathrm{mg} / \mathrm{ml}$ (volume fraction of $0-110 \%$, i.e., varying from the very dilute to the entangled regime). The samples were shaken vigorously in order to obtain a homogeneous dispersion. As soon as the shak-

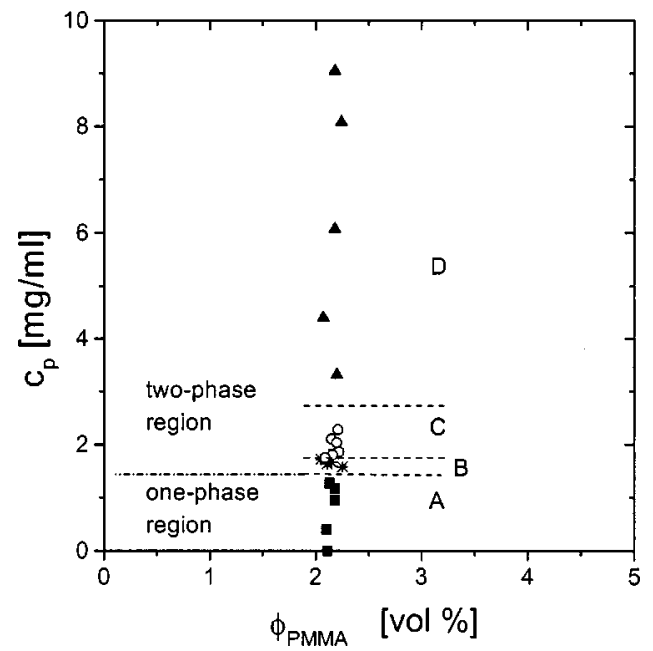

FIG. 3. Phase diagram of the colloid-polymer suspension with the four regimes indicated.

ing was stopped, phase separation started immediately for the samples within a certain polymer concentration range. It took about $30 \mathrm{sec}$ before the first image could be taken with the microscope. The phase separation was reversible in the sense that by shaking the sample became homogeneous again.

Four different regimes were found in the phase diagram as indicated in Fig. 3. Regime $A$ is the one-phase region $\left(c_{p}\right.$ $<1.28 \mathrm{mg} / \mathrm{ml}$ ) and regimes $B-D$ are in the two-phase region $\left(c_{p}>1.58 \mathrm{mg} / \mathrm{ml}\right)$. Figure 4 shows time series of $x y$ scans of the first hour after homogenization at a position of $20 \mu \mathrm{m}$ from the lower wall, and Fig. 5 shows $x z$ scans after an hour and $x y$ scans after several days. Representative series of the various regimes are given. The time is in units of the Brownian time $\tau_{B}$, defined as

$$
\tau_{B}=\frac{R^{2}}{D}=\frac{6 \pi \eta R^{3}}{k_{B} T},
$$

with $R$ the radius of the PMMA particles, $D$ the diffusion coefficient, $\eta$ the viscosity of the solvent with the polymer, $k_{B}$ the Boltzmann constant, and $T$ the temperature.

\section{One-phase region}

Regime $A\left(0<c_{p}<1.28 \mathrm{mg} / \mathrm{ml}\right)$ is the one-phase region; the polymer concentration is so low that no phase separation took place. Sedimentation occurs because of the small difference in density between the individual particles and the solvent. In about one day, a sediment was formed; the total height of the sediment was smaller than $1 \mathrm{~mm}$. The addition of a small amount of polymers increased the sedimentation rate. At low polymer concentration, the sediment was entirely crystalline within a day, whereas for a sample without polymers it took several days to weeks before the sediment was crystalline.

\section{Two-phase region}

In regime $B\left(1.58<c_{p}<1.72 \mathrm{mg} / \mathrm{ml}\right)$, the polymer concentration is just high enough for phase separation to occur. 


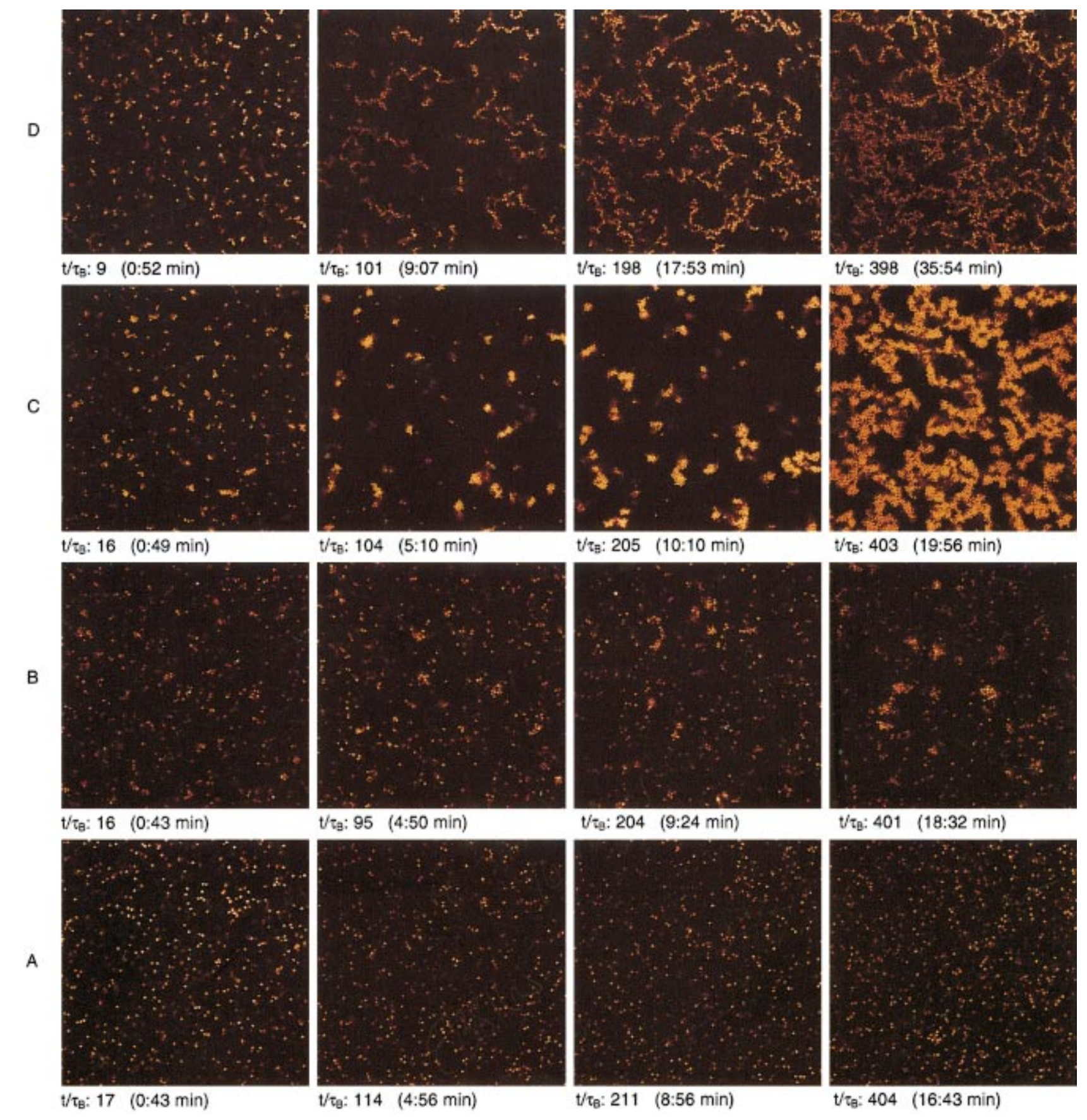

FIG. 4. (Color) Time series of CSLM images of $x y$ scans of the first hour after homogenization at a position of $20 \mu \mathrm{m}$ from the lower wall; image size $100 \mu \mathrm{m} \times 100 \mu \mathrm{m}$. Top row: regime $D, \phi=2.24 \%, c_{p}=8.07 \mathrm{mg} / \mathrm{ml}$; second row: regime $C, \phi=2.15 \%, c_{p}=2.10$ $\mathrm{mg} / \mathrm{ml}$; third row: regime $B, \phi=2.05 \%, c_{p}=1.72 \mathrm{mg} / \mathrm{ml}$; fourth row: regime $A, \phi=2.18 \%, c_{p}=1.18 \mathrm{mg} / \mathrm{ml}$.

The mechanism is like nucleation and growth; the clusters formed seem to be of finite size (although not entirely crystalline) in a sea of monomers. Rapid sedimentation of these clusters is observed. The growth of a single cluster could not easily be followed because of the high sedimentation rate of the clusters. The total number of single particles is only slightly decreased at the intermediate stage. The sediment is polycrystalline and the crystallites seem to have originated from the initial clusters. Macroscopically, Bragg reflections in white light were observed in this sediment, also indicating the presence of crystals.

In regime $C$, at intermediate polymer concentration $\left(1.75<c_{p}<2.28 \mathrm{mg} / \mathrm{ml}\right)$, aggregates are formed from single particles followed by growth of the clusters by aggregation. The clusters are not crystalline, but they are dense. After a while, sedimentation is observed by the increase of the number of particles in the image. The sediment formed is dense and no crystallinity was observed, although there seem to be 


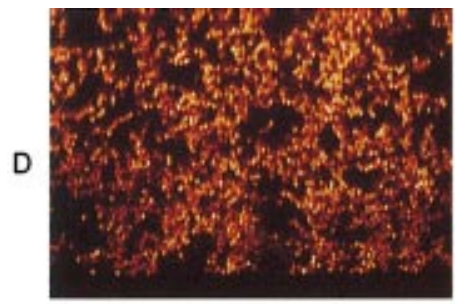

$x z ; 77: 11 \mathrm{~min}$

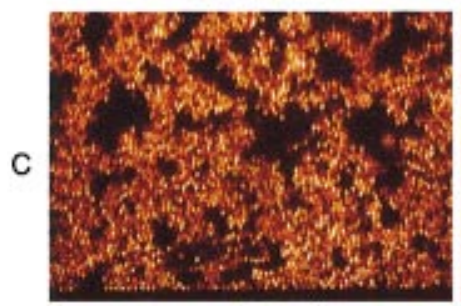

$x z ; 42: 25 \mathrm{~min}$

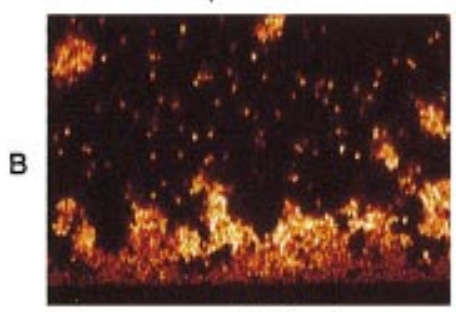

$x z ; 40: 51 \mathrm{~min}$

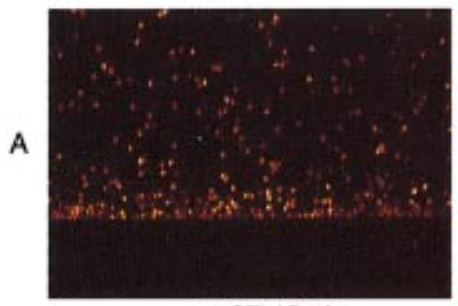

$x z ; 27 ; 45 \mathrm{~min}$

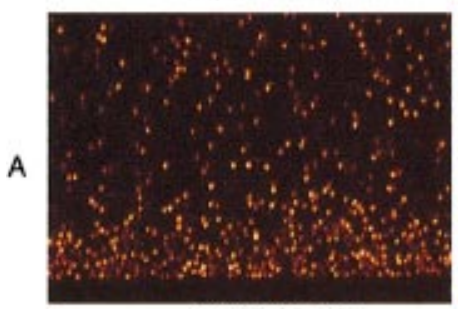

$x z ; 40: 00 \mathrm{~min}$

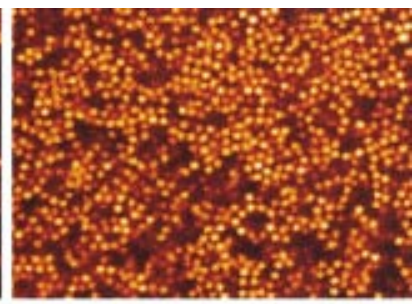

xy; 3 days

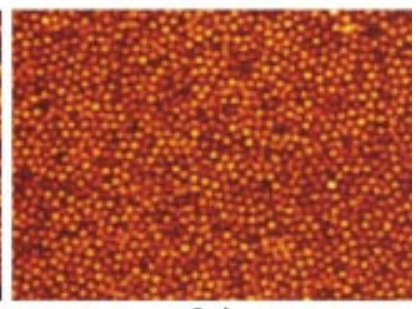

xy; 3 days

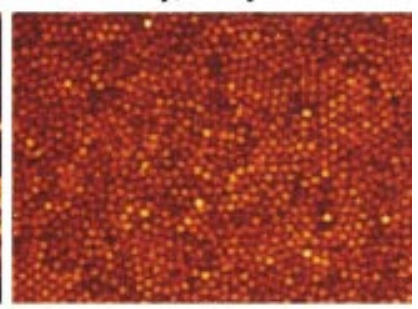

$\mathrm{xy} ; 2$ days

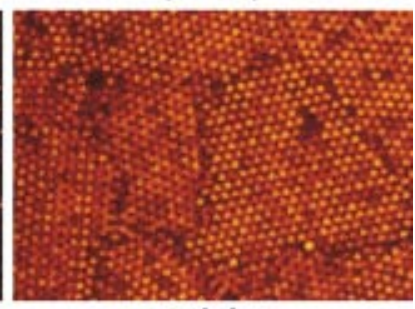

xy; 1 day

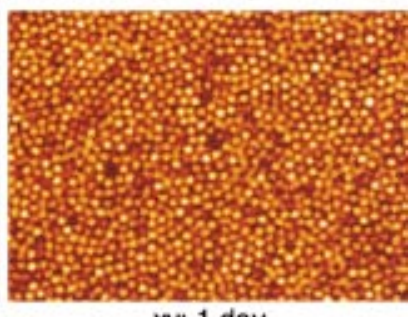

$x y ; 1$ day
FIG. 5. (Color) CSLM images of $x z$ scans after an hour and of $x y$ scans after several days. Top row: regime $D, \phi=2.24 \%, c_{p}$ $=8.07 \mathrm{mg} / \mathrm{ml}$; second row: regime $C, \phi=2.15 \%, c_{p}$ $=2.10 \mathrm{mg} / \mathrm{ml}$; third row: regime $B, \phi=2.05 \%, c_{p}=1.72 \mathrm{mg} / \mathrm{ml}$; fourth row: regime $A, \phi=2.18 \%, c_{p}=1.18 \mathrm{mg} / \mathrm{ml}$; fifth row: regime $A, \phi=2.11 \%, c_{p}=0 \mathrm{mg} / \mathrm{ml}$.

areas with short-ranged ordering.

In regime $D\left(3.31<c_{p}<9.04 \mathrm{mg} / \mathrm{ml}\right)$, aggregation is also observed, but here the clusters have a more ramified or elongated stringlike shape. Sedimentation is observed, and the sediment formed is dilute, getting denser in a few days. No crystallinity is observed in the sediment. The highest polymer concentration used here $(9.04 \mathrm{mg} / \mathrm{ml})$ is slightly above the overlap concentration $\left(c^{*}=8.15 \mathrm{mg} / \mathrm{ml}\right)$, but no difference in morphology compared with the behavior below $c^{*}$ was observed.

\section{ANALYSIS}

The formation of the clusters is analyzed quantitatively by digital image processing determining the coordinates of all the particles in each time frame, using procedures similar to the ones described by Crocker and Grier [24]. The confocal images are $2 \mathrm{D}$ cross sections of the sample in time at a certain position. In fact, they are $2 \mathrm{D}$ projections of a slab with a thickness of approximately $0.6 \mu \mathrm{m}$ (equal to the radius of the colloids), determined by the axial resolution of the microscope. Within this thin slab, it is not possible to distinguish between particles that are in focus and the ones that are slightly out of focus. In general, the latter ones have lower intensity, but particles that have been in focus for some time are bleached and therefore will have a lower intensity as well. As it is impossible to distinguish between the particles in focus and the ones slightly out of focus within this small slab, all particles were taken into account in the following analysis. For these reasons, it is not possible to analyze a radial distribution function or local bond-order parameter.

Figure 6 shows some of the results of the image analysis in four graphs. Here, the time scales are again normalized by the Brownian time, $\tau_{B}$. In the analysis, a cluster criterion is used to define the maximum distance between particle centers in order to be of the same cluster. This distance is chosen equal to the position of the first minimum in the projected $2 \mathrm{D} g(r)$ [25], which was determined for each sample at $t / \tau_{B}=600$. We do not expect the projection "error" to influence our results too much [26]. Figure $6(A)$ gives the total number of particles, $N_{\text {tot }}$, present in each time frame. In the initial stage $N_{\text {tot }}$ remains constant, followed by an increase due to sedimentation for the higher polymer concentration regions. Several plateaus at the ascending slopes are observed, suggesting that the densification or rearrangement of the sediment is not a continuous process. In Fig. $6(B)$, the total number of single particles normalized by the total number of particles per frame, $N_{\text {single }} / N_{\text {tot }}$, is given as a function of time; this gives information about the aggregation rate. We observe a very different dependence on time for the three mechanisms in the phase-separating region. At the highest polymer concentration (regime $D$ ), the aggregation rate is the highest, followed by the intermediate polymer concentration (regime $C$ ). At the lowest polymer concentration (regime $B$ ), the number of single particles remains high for a long time compared to the other two regions. Finally, we see a decrease in this curve due to the growth of the layer of sedimented clusters. Figure $6(C)$ gives the averaged number of particles per cluster, $\left\langle N_{c}\right\rangle$, as a function of time. Single particles are not considered as clusters of one particle in this analysis. The plateaus from Fig. 6(A) are recovered in this graph. Initially, the elongated clusters of regime $D$ contain more particles than the more compact ones of regime $C$. At intermediate times, the compact clusters of regime $C$ contain more particles. The difference between the initial and intermediate stages is caused by the difference in aggregation rate. In regime $D$, the aggregation rate is higher than in regime $C$. This means that the number of particles that are part of a cluster is increasing faster in regime $D$. When most of the single particles are part of a cluster, then the average number 

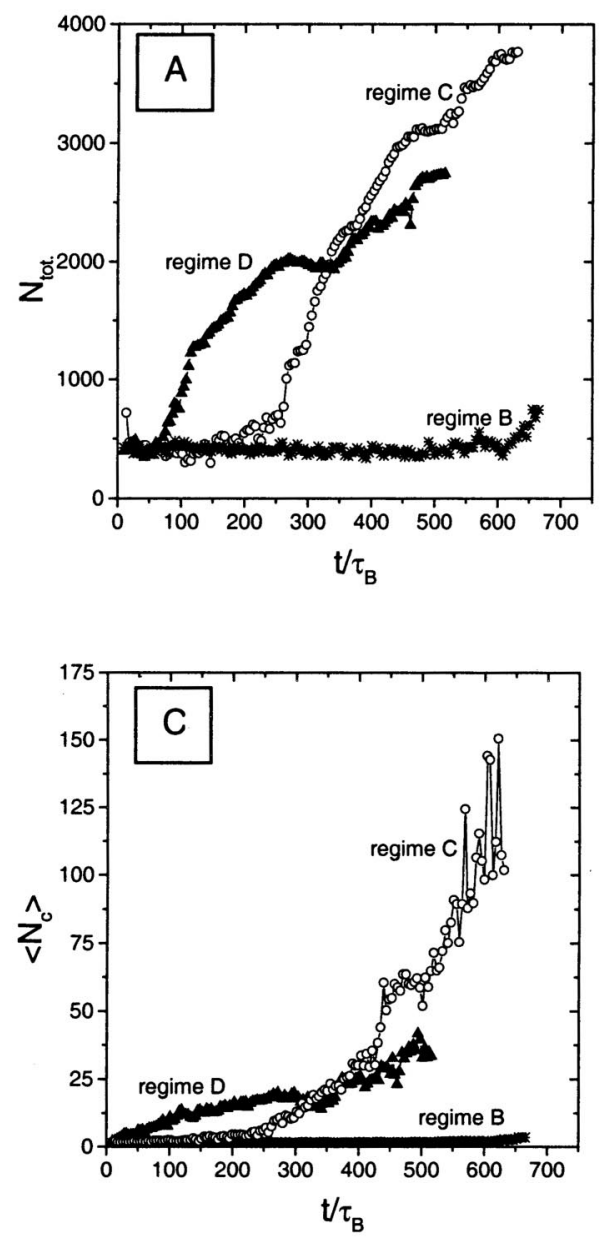

of particles per cluster is no longer influenced by the difference in aggregation rate, but only the result of the morphology of the clusters. In regime $B$, at the lowest polymer concentration, the average number remains low. Figure $6(D)$ gives the average number of neighbors per particle in a cluster, $\left\langle N_{n}\right\rangle$. Initially, the particles in the elongated clusters of regime $D$ have more neighbors than the more compact ones of regime $C$, again due to the higher aggregation rate. At intermediate times, $\left\langle N_{n}\right\rangle$ is higher for the compact clusters, so these are becoming denser than the elongated clusters. In regime $B$, the number of neighbors remains low for a long time. The increase starts at the end of the curve. By this time, more clusters formed are observed in one time frame because of sedimentation.

The rate of aggregation can be analyzed in further detail by a comparison with the theory of Smoluchowski for aggregation [27]. It is assumed that only the Brownian motion of the particles determines the process, and when particles collide they will stick together. When we start with a number density of particles $n_{0}$, the decrease of the number of single particles, $N_{\text {single }}$, is described by

$$
\frac{N_{\text {single }}}{V}=\frac{\eta_{0}}{\left(1+t / \tau_{B}\right)^{2}}
$$

with $\tau_{S}=\left(8 \pi D R n_{0}\right)^{-1}, \quad D=k_{B} T / 6 \pi \eta R, \quad$ and $n_{0}$ $=3 \phi / 4 \pi R^{3}$.
FIG. 6. Results of digital image analysis. $A$ : the total number of particles, $N_{\text {tot }}$, present in each time frame; $B$ : the total number of single particles normalized by the total number of particles per frame, $N_{\text {single }} / N_{\text {tot }}$, as a function of time; $C$ : the averaged number of particles per cluster, $\left\langle N_{c}\right\rangle$, as a function of time; $D$ : the average number of neighbors per particle in a cluster, $\left\langle N_{n}\right\rangle$.
In this equation, $t$ is the time, $\tau_{S}$ is the Smoluchowski time, $D$ is the diffusion coefficient, $k_{B}$ is the Boltzmann constant, $T$ is the temperature, $\eta$ is the viscosity, $R$ is the radius of the particle, and $\phi$ is the volume fraction of the particles. This equation is used only for the initial stage of the experiments. The initial stage is defined as the time period in which the total number of particles observed in the confocal images remains constant, using Fig. 6(a). The original number density of particles, $n_{0}$, is determined from the total number of particles observed in the confocal images of the initial stage, and the number of single particles per time frame is determined using the cluster criterion, as defined above. The Smoluchowski time $\tau_{S}$ is now determined by a fit of the plot of the number of single particles per time frame as a function of time for the initial stage, using Eq. (2). In comparison, the Smoluchowski time could be calculated using the definitions given below Eq. (2). The viscosity is a function of the polymer concentration, which was measured with a rheometer.

In Fig. 7, we compare the Smoluchowski time predicted from theory with the one determined by fitting the data obtained by digital image processing. At high polymer concentration (regime $D$, formation of elongated clusters), the calculated and the determined value follow the same trend and are of the same order of magnitude. However, going to the lower polymer concentrations (regime $C$, formation of compact clusters), a sudden, rapid increase in the determined value is observed, while the calculated value is still decreas- 


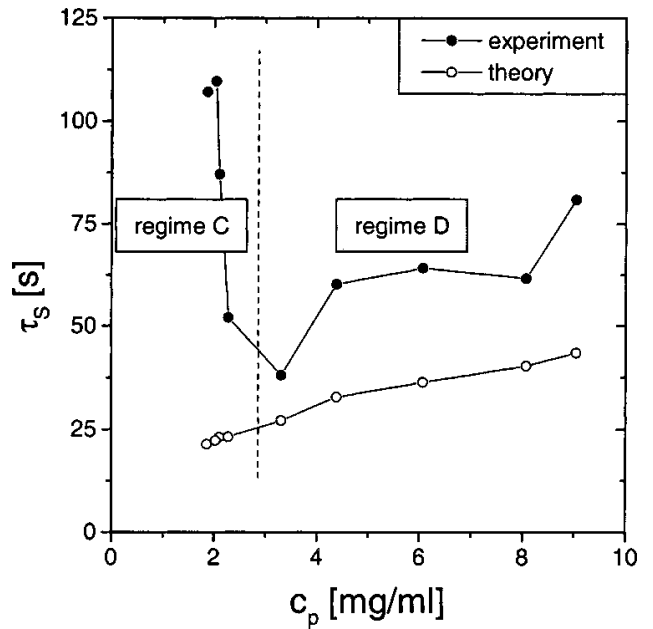

FIG. 7. The predicted and determined Smoluchowski times.

ing. So the aggregation process in regime $C$ cannot be described by the Smoluchowski equation for rapid coagulation.

\section{DISCUSSION}

It is possible to distinguish three stages in the phaseseparation process: the initial stage in which sedimentation is not observed, the intermediate stage in which sedimentation plays a role (observed as an increase of particles in the CSLM image), and the final stage in which the sediment has reached its final density. The morphology of the sediments in these experiments is not only determined by the induced attractions, but also by the gravitational field. Sedimentation alters the concentration of particles, which has in turn an influence on the kinetics of the phase separation in the intermediate stage, e.g., with respect to collision and bonding probabilities.

The CSLM experiments explain the difficulty in finding the phase boundary by visual inspection. Just across the phase boundary we found with confocal microscopy the nucleationlike mechanism (regime $B$ ), where the number of single particles remains high for a long time. The sedimentation rate of such a phase-separating sample is not much different from that of a sample in the one-phase region near the phase boundary. Therefore, it is difficult to distinguish a phase-separating suspension from a non-phase-separating one near the phase boundary.

In the one-phase region, the structure of the sediment formed in the sedimentation tubes was different from the ones observed in the confocal vials. Actually, the effect of the polymer concentration on the structure was opposite in the two types of sample containers. In the sedimentation tubes, the addition of polymers decreased the amount of crystals, whereas in the confocal vials the addition of polymers increased the formation of crystals. It appears that the structure of the sediment is not only dependent on the concentrations, but also on the form and size of the tubes and the amount of dispersion. It is a complex interplay between several parameters that determines the final structure of the sediment [28]. In these experiments, the most important difference between the two types of sample containers is probably the total amount of the sediment formed. In the sedimentation tubes it is about $1 \mathrm{~cm}$ and in the confocal vials it is less than $1 \mathrm{~mm}$. This implies that the pressure exerted on the particles due to the height of the sediment is different in the two kinds of sample containers, on the order of 100 times. In the larger, sedimentation tubes, this higher pressure might prevent crystallization. On the other hand, the small attraction between the colloidal particles, induced by the addition of only a small amount of polymers, might enhance crystallization. This may explain why in the confocal vials the sediment of the samples with a small amount of polymers added crystallizes more easily compared to that without any polymer.

In the phase-separating region, we found three regimes with very different characteristics. Not only the kinetics of the phase separation, but also the morphology of the resulting sediments differs. The boundaries between these three regimes are surprisingly narrow.

Regime B, which is very narrow, is clearly distinguished from regimes $C$ and $D$ by the nucleationlike nature of the mechanism and the high number of single particles throughout the initial and intermediate stage. Regimes $C$ and $D$ are distinguished from one another by the morphology of the clusters and the aggregation rate. The structure of the clusters in regime $D$ is open and stringlike, while in regime $C$ it is denser, more compact, and seems to have some ordering. The transition from regime $C$ to $D$ is also indicated by the determination of the Smoluchowski times in the initial stage. Regime $D$ can be described by the Smoluchowski equation for rapid aggregation, whereas in regime $C$ there is a discrepancy between the determined and expected value for the Smoluchowski times. We could consider regime $D$ as an "irreversible" diffusion-limited cluster aggregation (DLCA), only determined by the diffusion of the particles and irreversible in the sense that once particles encounter they will stick together. Analogously, regime $C$ can be considered as a "reversible" DLCA; particles encounter but there is a probability less than 1 for the particles to stick together. The "reversible" DLCA is sometimes also termed reactionlimited cluster aggregation (RLCA).

Brownian dynamics simulations allow a direct comparison with real-space experiments (e.g., [29]). A recent Brownian dynamics simulation on dilute systems with short-ranged attractive interactions [30] shows striking similarities with the confocal images described here. Three regimes were obtained with morphology similar to the ones we found experimentally in the colloid-polymer suspensions.

The kinetics of phase separation have already been extensively studied by light scattering [8,31-34]. Spinodal-like behavior was found in various studies. The main issue here seems to be the subtle boundaries between spinodal decompositionlike behavior and DLCA [33]. We believe that with confocal microscopy we have a tool at hand to solve this matter, because we can study bulk processes on a particle level.

Poon et al. [8] defined various regimes of phase behavior using light scattering, which we will compare here to our results. Poon et al. distinguished three regions: a region where no phase separation occurs, a region of equilibrium 


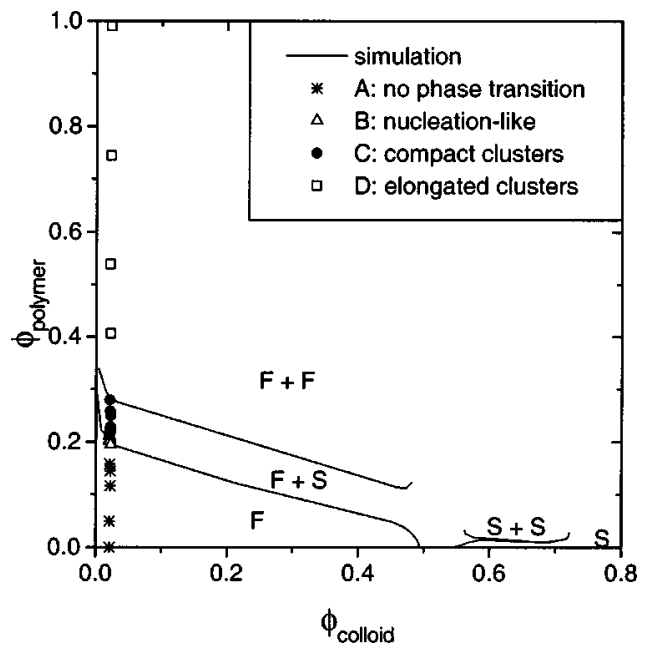

FIG. 8. Phase diagram obtained by simulation [9] with the experimental data points indicated.

phase transition, and a region of nonequilibrium phase transition. In the last region, three mechanisms were found: a nucleationlike behavior, a spinodal-like behavior, and transient gelation. The nonequilibrium phase boundary is determined by a difference in sedimentation rate of the solid phase. Our regime $B$ has the same characteristics as in two regions defined by Poon et al.: the equilibrium transition region and the nonequilibrium region with the nucleationlike behavior. According to Poon et al., there should be a subtle change in sedimentation rate only, which is not easy to observe in the small samples that we used. Furthermore, we did not observe a region defined as transient gelation. However, this is expected, because we worked at a very low colloid concentration. The most interesting region to compare is the region where Poon et al. found a spinodal-like behavior. It is called spinodal-like behavior because at intermediate times the scattering exhibits dynamic scaling with the exponent $\gamma$ $=3$, the spatial dimension. This behavior resembles that of spinodal decomposition. At higher polymer concentrations, the dynamic scaling still holds but the exponent is reduced to 1.8, which is equal to the fractal dimension of aggregates formed by DLCA. Furthermore, they found the structure of these samples under the microscope to be interconnected domain patterns. We found in this region two regimes, $C$ and $D$. We defined them both as a DLCA behavior in the initial stage (when no sedimentation is observed), but we made a distinction between reversible DLCA in regime $\mathrm{C}$ and irreversible DLCA in regime $D$. In both cases, an interconnected structure is observed after some time. However, it originates not from spinodal decomposition but from aggregation of the particles and the effect of sedimentation.

Poon et al. connect the various regimes in phase behavior to the phase diagram. They suggest that nonequilibrium behavior is related to metastable phase boundaries [35]. We compare our results of the various regimes with a phase diagram obtained by simulations for a model colloid-polymer mixture with the size ratio of polymer to colloid of 0.1 by Dijkstra et al. [9]. Figure 8 shows the phase diagram with the fluid-solid phase boundary and the phase boundaries for the metastable fluid-fluid and solid-solid phase transitions adapted from Dijkstra et al. [9]. In the same figure, the samples investigated by CSLM are indicated and the various regimes are specified. We find that the fluid-solid phase boundary coincides with the results of our experiments. Furthermore, regime $B$ and regime $C$ are between the fluid-solid binodal and the metastable fluid-fluid boundary. We do not know if the position of these two regimes is influenced by the metastable boundary or whether it is a coincidence.

A narrow region of optimal crystallization is reported for crystallization of globular proteins as well [36], where aggregates leading to crystallization are called CRAGG's (crystallizing aggregates) and aggregates leading to amorphous sediments are called PRAGG's (precipitating aggregates). The distinction between a CRAGG and a PRAGG at an early stage is important. If we make the comparison with our experiments, we see that in regime $B$ (low polymer concentration; nucleationlike mechanism), CRAGG's are formed, resulting in this case in a polycrystalline sediment. Regime $B$ can now easily be recognized in the initial stage by the existence of a few large clusters in a sea of single particles, whereas in regimes $C$ and $D$, first almost all the single particles aggregate to small clusters, which in turn aggregate to bigger clusters, resulting in PRAGG's. From the images of the initial stages of the three mechanisms, it was not clear at first that only regime $B$ results in a crystalline sediment. Also the clusters in regime $C$ are dense and seem to have some ordering, but the resulting sediment is not crystalline. Sedimentation in this case could be the reason that the clusters were prevented from crystallizing.

\section{CONCLUSIONS}

The combination of fluorescence confocal scanning laser microscopy and a colloidal suspension with some specific characteristics allows us to study bulk phase separation in real space and real time on a particle level. Three mechanisms for phase separation in a colloid-polymer suspension were found as a function of the polymer concentration (i.e., strength of attraction between the colloidal particles): regime $B$ (very narrow), a nucleationlike mechanism at low polymer concentrations; regime $C$, aggregation leading to compact clusters (reaction-limited aggregation) at intermediate polymer concentrations; regime $D$, aggregation leading to elongated clusters (diffusion-limited aggregation) at higher polymer concentrations. The borders between the three regimes are quite sharp. Digital image processing was used to characterize the three mechanisms semiquantitatively. The final structure of a phase-separating sample is determined by the mechanism of phase separation in the initial stage and the influence of the gravitational field in the intermediate stage. Moreover, we found that the size and the shape of the sample container have an effect on the final structure as well. Only in regime $B$, at low polymer concentration, did the sediment contain crystalline parts. However, in regime $C$, at intermediate polymer concentration, there seemed to be some ordering at short range. It is possible that the gravitational field prevents the system from forming a crystalline sediment. In regime $D$, at high polymer concentrations, no ordering was found in the final structure. 


\section{ACKNOWLEDGMENTS}

We thank Gilles Bosma for the synthesis of the PMMA particles, Valerie Anderson for valuable discussions, and Marjolein Dijkstra for providing us with the calculated phase diagram. This work was supported by the Stichting voor Fundamental Onderzoek der Materie (Foundation for Fundamental Research on Matter), which is part of the Nederlandse Organisatie voor Wetenschappelijk Onderzoek (Netherlands Organization for Advancement of Research).
[1] S. Asakura and F. Oosawa, J. Chem. Phys. 22, 1255 (1954).

[2] A. Vrij, Pure Appl. Chem. 48, 471 (1976).

[3] A. P. Gast, C. K. Hall, and W. B. Russel, J. Colloid Interface Sci. 96, 251 (1983).

[4] H. N. W. Lekkerkerker, W. C. K. Poon, P. N. Pusey, A. Stroobants, and P. B. Warren, Europhys. Lett. 20, 559 (1992).

[5] P. R. Sperry, J. Colloid Interface Sci. 99, 97 (1984).

[6] F. Leal Calderon, J. Bibette, and J. Bias, Europhys. Lett. 23, 653 (1993).

[7] S. M. Ilett, A. Orrock, W. C. K. Poon, and P. N. Pusey, Phys. Rev. E 51, 1344 (1995).

[8] W. C. K. Poon and M. D. Haw, Adv. Colloid Interface Sci. 73, 71 (1997).

[9] M. Dijkstra, J. M. Brader, and R. Evans, J. Phys.: Condens. Matter 11, 10079 (1999).

[10] P. N. Pusey, A. D. Pirie, and W. C. K. Poon, Physica A 201, 322 (1993).

[11] N. A. M. Verhaegh, D. Asnaghi, H. N. W. Lekkerkerker, M. Giglio, and L. Cipelletti, Physica A 242, 104 (1997).

[12] A. Kose and S. Hachisu, J. Colloid Interface Sci. 55, 487 (1976).

[13] C. Smits, B. van der Most, J. K. G. Dhont, and H. N. W. Lekkerkerker, Adv. Colloid Interface Sci. 42, 33 (1992).

[14] E. K. Hobbie and M. J. Holter, J. Chem. Phys. 108, 2618 (1998)

[15] D. J. Robinson and J. C. Earnshaw, Phys. Rev. Lett. 71, 715 (1993).

[16] A. van Blaaderen and P. Wiltzius, Science 270, 1177 (1995).

[17] W. K. Kegel and A. van Blaaderen, Science 287, 290 (2000).

[18] E. R. Weeks, J. C. Crocker, A. C. Levitt, A. Schofield, and D. A. Weitz, Science 287, 627 (2000).
[19] A. van Blaaderen, R. Ruel, and P. Wiltzius, Nature (London) 385, 321 (1997).

[20] L. Antl, J. W. Goodwin, R. D. Hill, R. H. Ottewil, S. M. Owens, and S. Papworth, Colloids Surface 17, 67 (1986).

[21] G. Bosma, C. Pathmamanoharan, E. H. A. de Hoog, W. K. Kegel, A. van Blaaderen, and H. N. W. Lekkerkerker (unpublished).

[22] P. Debye and A. N. Bueche, J. Chem. Phys. 16, 573 (1948).

[23] E. H. A. de Hoog, G. Bosma, W. K. Kegel, A. van Blaaderen, and H. N. W. Lekkerkerker (unpublished).

[24] J. C. Crocker and D. G. Grier, J. Colloid Interface Sci. 179, 298 (1996).

[25] P. R. ten Wolde and D. Frenkel, J. Chem. Phys. 109, 9901 (1998).

[26] K. S. Rao and R. Rajagopalan, Phys. Rev. E 57, 3227 (1998).

[27] M. von Smoluchowski, Z. Phys. Chem., Stoechiom. Verwandtschaftsl. 92, 129 (1917).

[28] J. K. G. Dhont, in An Introduction to Dynamics of Colloids, edited by D. Möbius and R. Miller (Elsevier, Amsterdam, 1996), Chap. 7.

[29] K. G. Soga, J. R. Melrose, and R. C. Ball, J. Chem. Phys. 108, 6026 (1998).

[30] G. A. Vliegenthart and E. H. A. de Hoog (unpublished).

[31] M. Carpineti and M. Giglio, Phys. Rev. Lett. 68, 3327 (1992).

[32] J. Bibette, T. G. Mason, H. Gang, and D. A. Weitz, Phys. Rev. Lett. 69, 981 (1992).

[33] P. Poulin, J. Bibette, and D. A. Weitz, Eur. Phys. J. B 7, 277 (1999).

[34] P. W. Rouw, A. T. J. M. Woutersen, B. J. Ackerson, and C. G. de Kruif, Physica A 156, 876 (1989).

[35] W. C. K. Poon, A. D. Pirie, and P. N. Pusey, Faraday Discuss. 101, 65 (1995).

[36] G. Feher and Z. Kam, Methods Enzymol. 114, 77 (1985). 